\title{
Anisotropic Turbulent Advection of a Passive Vector Field: Effects of the Finite Correlation Time
}

\author{
N. V. Antonov ${ }^{1, a}$ and N. M. Gulitskiy ${ }^{1, b}$ \\ ${ }^{1}$ Chair of High Energy Physics and Elementary Particles, Department of Theoretical Physics, Faculty of \\ Physics, Saint Petersburg State University, Ulyanovskaja 1, Saint Petersburg-Petrodvorez, 198504 Russia
}

\begin{abstract}
The turbulent passive advection under the environment (velocity) field with $f$ nite correlation time is studied. Inertial-range asymptotic behavior of a vector (e.g., magnetic) field, passively advected by a strongly anisotropic turbulent flow, is investigated by means of the field theoretic renormalization group and the operator product expansion. The advecting velocity field is Gaussian, with finite correlation time and prescribed pair correlation function. The inertial-range behavior of the model is described by two regimes (the limits of vanishing or infinite correlation time) that correspond to nontrivial fixed points of the RG equations and depend on the relation between the exponents in the energy spectrum $\mathcal{E} \propto k_{\perp}^{1-\xi}$ and the dispersion law $\omega \propto k_{\perp}^{2-\eta}$. The corresponding anomalous exponents are associated with the critical dimensions of tensor composite operators built solely of the passive vector field itself. In contrast to the well-known isotropic Kraichnan model, where various correlation functions exhibit anomalous scaling behavior with infinite sets of anomalous exponents, here the dependence on the integral turbulence scale $L$ has a logarithmic behavior: instead of power-like corrections to ordinary scaling, determined by naive (canonical) dimensions, the anomalies manifest themselves as polynomials of logarithms of $L$. Due to the presence of the anisotropy in the model, all multiloop diagrams are equal to zero, thus this result is exact.
\end{abstract}

\section{Introduction}

Understanding the turbulent advection is a rich and challenging problem. On the one hand, the violation of the classical Kolmogorov-Obukhov theory [1] is even more strongly pronounced for a advected field than for the velocity field itself; see, e.g., review [2]; on the other hand, the problem of passive advection appears to be easier tractable theoretically. The most remarkable progress on this way was achieved for the so-called Kraichnan's rapid-change model [3], in which instead of the stochastic Navier-Stokes (NS) equation the velocity field is modeled by a Gaussian ensemble, not correlated in time, with zero mean and prescribed pair correlation function. This approximation corresponds to the passive field approximation: if we neglect the influence of advected field $\boldsymbol{\theta}$ to the dynamics of the environment (velocity) field $\mathbf{v}$, the latter can be modeled by statistical ensembles with prescribed properties. For the first time, the anomalous exponents have been calculated on the basis of a microscopic model and within regular expansions in formal small parameters in [2].

\footnotetext{
ae-mail: n.antonov@spbu.ru

be-mail: n.gulitskiy@spbu.ru
} 
In this paper, we consider a more realistic model with finite (and not small) correlation time. The passively advected field is chosen as a vector and corresponds to the magnetohydrodynamic (MHD) turbulence. Furthermore, we will investigate the "strongly anisotropic" model, which is obtained by introducing the velocity field $\mathbf{v}$, oriented along a fixed direction $\mathbf{n}$ :

$$
\boldsymbol{v}(t, \mathbf{x})=\mathbf{n} \times v\left(t, \mathbf{x}_{\perp}\right) .
$$

This problem is closely related to the processes taken place in solar corona, e.g., with solar wind [4], and $\mathbf{n}$ is an "orientation of a large-scale flare" in this context. As well, this model can be viewed as a $d$-dimensional generalization of the strongly anisotropic velocity ensemble introduced in [5] in connection with the turbulent diffusion problem and further studied and generalized in a number of papers [6-8]. Following [9], we included into the stochastic advection-diffusion equation [see equation (2) below] an additional arbitrary dimensionless parameter $\mathcal{A}_{0}$, which unifies different physical situations: the kinematic MHD model, the linearized NS equation and the passive admixture with complex internal structure of the particles.

One of the most convenient ways to study the anomalous scaling in various statistical models of the turbulent advection is to apply the field theoretic renormalization group (RG) and operator product expansion (OPE); see, e.g., the monographs [10,11]. In this case the anomalous scaling is a consequence of the existence in the model of composite fields ("composite operators" in the quantumfield terminology) with negative scaling dimensions; see [12,13] and references therein.

This model should be considered as generalization and evolution of the models, considered in [7], where the advected field is chosen scalar, and [14], where the environment (velocity) field is chosen decorrelated in time. The main result of the present paper is that the inertial-range behavior of vector fields advected by velocity ensemble with finite correlation time combines the features of both above models: as in the scalar case, there is a set of fixed points, governing the infrared (IR) behavior; as in the zero-time correlation model, the inertial-range behavior of vector fields has logarithmic corrections to ordinary scaling.

\section{Description of the model}

In the presence of the anisotropy, the turbulent advection of a passive vector field $\boldsymbol{\theta}(x) \equiv \boldsymbol{\theta}(t, \mathbf{x})$ is described by the stochastic equation [14]

$$
\partial_{t} \theta_{i}+\partial_{k}\left(v_{k} \theta_{i}-\mathcal{A}_{0} v_{i} \theta_{k}\right)+\partial \mathcal{P}=v_{0}\left(\partial_{\perp}^{2}+f_{0} \partial_{\|}^{2}\right) \theta_{i}+f_{i},
$$

where $\theta_{i}(x)$ is the vector field, $x \equiv\{t, \mathbf{x}\}, \partial_{t} \equiv \partial / \partial t, \partial_{i} \equiv \partial / \partial x_{i}, \mathbf{n}$ is a unit vector that determines the distinguished direction, $\mathbf{x}_{\perp}$ and $\partial_{\perp}$ are the components of the vectors $\mathbf{x}$ and $\boldsymbol{\partial}$ perpendicular to $\mathbf{n}$, $\partial_{\|} \equiv \boldsymbol{\partial} \cdot \mathbf{n}, v_{0}$ is the molecular diffusivity coefficient, $\partial^{2}$ is the Laplace operator, $\boldsymbol{v}(x) \equiv\left\{v_{i}(x)\right\}$ is the velocity field, $f_{i} \equiv f_{i}(x)$ is an artificial Gaussian scalar noise with zero mean and correlation function

$$
\left\langle f_{i}(t, \mathbf{x}) f_{k}\left(t^{\prime}, \mathbf{x}^{\prime}\right)\right\rangle=\delta\left(t-t^{\prime}\right) C_{i k}(\mathbf{r} / L)
$$

Here $\mathbf{r}=\mathbf{x}-\mathbf{x}^{\prime}, r=|\mathbf{r}|$, the parameter $L \equiv M^{-1}$ is the integral (external) turbulence scale related to the stirring, and $C_{i k}$ is a dimensionless function finite for $r / L \rightarrow 0$ and rapidly decaying for $r / L \rightarrow \infty$. The dimensionless constant $f_{0}$, which breaks the $O_{d}$ symmetry of the Laplace operator, had to be introduced for renormalizability reasons.

As we consider the passive field approximation, the field $\boldsymbol{v}(x)$ can be simulated by a statistical ensemble with prescribed statistics: it is assumed to be Gaussian, strongly anisotropic, homogeneous, 
and with zero mean and a correlation function [7]

$$
\left\langle v_{a}(t, \mathbf{x}) v_{a^{\prime}}\left(t^{\prime}, \mathbf{x}^{\prime}\right)\right\rangle=2 \pi n_{a} n_{a^{\prime}} \int_{k>m} \frac{d \mathbf{k}}{(2 \pi)^{d}} \delta\left(k_{\|}\right) e^{i \mathbf{k} \cdot\left(\mathbf{x}-\mathbf{x}^{\prime}\right)} D_{0} \frac{k_{\perp}^{5-d-(\xi+\eta)}}{\omega^{2}+\left[\alpha_{0} v_{0} k_{\perp}^{2-\eta}\right]^{2}} .
$$

Here $d$ is the dimensionality of the $\mathbf{x}$ space, $k_{\perp} \equiv\left|\mathbf{k}_{\perp}\right|, 1 / m$ is another integral turbulence scale, related to the stirring, $D_{0}>0$ is the amplitude factor and the symbol $k_{\|}$denotes the scalar product $\mathbf{k} \cdot \mathbf{n}$. Unlike [15] and some other generalizations of the isotropic Kraichnan's model, this velocity ensemble does not contain the isotropic case as a special case. The function (4) involves two independent exponents $\xi$ and $\eta$, which in the RG approach play the role of two formal expansion parameters; depending on their values, the model reveals various types of inertial-range scaling regimes (see Sec. 3). A new parameter $\alpha_{0}$ is needed for dimensionality reasons.

\section{Field theoretic formulation of the model. Renormalization}

The stochastic problem (2) - (4) is equivalent to the field theoretic model of the extended set of three fields $\Phi \equiv\left\{\boldsymbol{\theta}, \boldsymbol{\theta}^{\prime}, \boldsymbol{v}\right\}$ with the action functional

$$
\mathcal{S}(\Phi)=-\frac{1}{2} v_{i} D_{v}^{-1} v_{k}+\frac{1}{2} \theta_{i}^{\prime} D_{\theta} \theta_{k}^{\prime}+\theta_{k}^{\prime}\left[-\partial_{t} \theta_{k}-\left(v_{i} \partial_{i}\right) \theta_{k}+\mathcal{A}_{0}\left(\theta_{i} \partial_{i}\right) v_{k}+v_{0}\left(\partial_{\perp}^{2}+f_{0} \partial_{\|}^{2}\right) \theta_{k}\right]
$$

Here all the terms, with the exception of the first one, represent the De Dominicis-Janssen action for the stochastic problem (2), (3) at fixed $\boldsymbol{v}$, while the first term represents the Gaussian averaging over $\boldsymbol{v}$. Furthermore, $D_{\theta}$ and $D_{v}$ are the correlators (3) and (4) respectively; the needed integrations over $x=(t, \mathbf{x})$ and summations over the vector indices are implied. This formulation means that the statistical averages of random quantities in the stochastic problem (2), (4) coincide with the functional averages of weight $\exp \mathcal{S}(\Phi)$ with the action (5).

The model (5) is solved by a standard Feynman diagrammatic technique with the triple vertex, which is represented in the diagrams as the point, in which three lines connect with each other, and the three bare propagators: $\left\langle v_{a} v_{a^{\prime}}\right\rangle_{0},\left\langle\theta_{c} \theta_{c^{\prime}}^{\prime}\right\rangle_{0}$ and $\left\langle\theta_{b} \theta_{b^{\prime}}\right\rangle_{0}$, which are determined by the quadratic (free) part of the action functional and are represented in the diagrams as wavy (which corresponds to the field $v$ ), slashed straight (the slashed end corresponds to the field $\theta^{\prime}$ ) and straight (the end without a slash corresponds to the field $\theta$ ) lines, respectively.

From the analysis of the ultraviolet (UV) divergences it follows, that we deal with two diverging functions: the response function $\left\langle\theta_{\alpha}^{\prime} \theta_{\beta}\right\rangle_{1-\text { ir }}$ and the triple correlation function $\left\langle\theta_{\alpha}^{\prime} \theta_{\beta} v_{\gamma}\right\rangle_{1-\text { ir }}$; but from direct calculations the latter appears to be equal to zero. Moreover, all multiloop diagrams entering into expansion of the 1-irreducible response function $\left\langle\theta_{\alpha}^{\prime} \theta_{\beta}\right\rangle_{1-i r}$, also are equal to zero, therefore to renormalize our model we have to calculate the only diagram represented in Fig. 1.

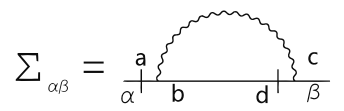

Figure 1. The only divergent diagram, entering into expansion of the 1-irreducible response function $\left\langle\theta_{\alpha}^{\prime} \theta_{\beta}\right\rangle_{1-\text { ir }}$

From the substitution of the explicit expression for the divergent part of the self-energy operator $\Sigma_{\alpha \beta}$ into the expression for the 1-irreducible linear response function $\left\langle\theta_{\alpha}^{\prime} \theta_{\beta}\right\rangle_{1-\text { ir }}$ it follows that the poles in $\xi$ and $\eta$ cannot be removed by the renormalization of the model parameters, and in order to ensure multiplicative renormalizability one has to add new term of the form $u_{0} f_{0} v_{0}\left(n_{k} \theta_{k}^{\prime}\right) \partial_{\|}^{2}\left(n_{k} \theta_{k}\right)$ into the action functional (5), with a new positive amplitude factor $u_{0}$. 
After this trick the model becomes multiplicatively renormalizable with two independent renormalization constants $Z_{f}$ and $Z_{u}$ :

$$
f_{0}=f Z_{f}, u_{0}=u Z_{u}, g_{0}=g \mu^{\xi+\eta} Z_{g} ; \quad \alpha_{0}=\alpha \mu^{\eta}, \quad \text { and } \quad Z_{g}=Z_{f}^{-1} .
$$

Here $\mu$ is the "reference mass" (additional free parameter of the renormalized theory) in the minimal subtraction (MS) renormalization scheme, and the coupling constant $g$ is defined as $g=D_{0} / v_{0}^{3} f_{0}$ with $D_{0}$ from (4).

One of the basic RG statements is that the asymptotic behavior of the model is governed by the fixed points $\left\{g_{i}^{*}\right\}$, defined by the relations

$$
\beta_{g}=\beta_{u}=\beta_{f}=\beta_{\alpha}=0
$$

the type of the fixed point (IR/UV attractive or a saddle point) is determined by the matrix $\Omega_{i k}=$ $\partial \beta_{i} / \partial g_{k}$ : for an IR attractive fixed point the matrix $\Omega$ has to be positive.

The analysis of the $\beta$-functions reveals four different IR attractive fixed points depending on the exponents $\xi$ and $\eta$ : if $\xi<0, \eta<0$, or if $\eta>0, \eta-\xi>0$, we have two gaussian fixed points with $g^{*}=0$ (regimes $1 a$ and $2 a$, respectively; see Fig. 2); if $\xi>0, \eta<0$, or if $\eta>0, \xi-\eta>0$, we have two nontrivial regimes, called $1 b$ and $2 b$, respectively; for detailes of the calculation see [16]. Thus we can conclude that the domains of IR stability in the vector model (5) coincide with the corresponding domains of IR stability in the scalar model considered in [7]. Since the $\beta$-functions have no higher-order corrections, this pattern is exact.

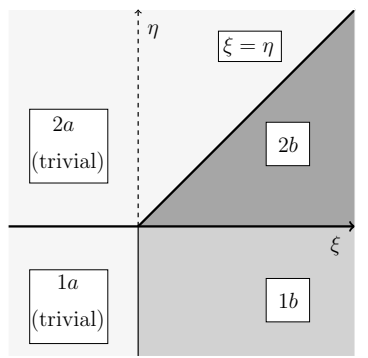

Figure 2. Domains of IR stability of the fixed points in the model (5). The numbers in boxes correspond to the fixed points $(1 a)-(2 b)$ in the text.

This fact implies that the correlation functions of the model (5) in the IR region ( $\mu r \simeq \Lambda r \gg 1$, $M r \sim 1$ ) exhibit scaling behavior (as we will see below, up to logarithmic factors). For the corresponding critical dimensions $\Delta[F] \equiv \Delta_{F}=d_{F}^{k}+2 d_{F}^{\omega}+\gamma_{F}^{*}$ we have

$$
\Delta_{t}=-\Delta_{\omega}=-2, \quad \Delta_{m}=\Delta_{M}=1, \quad \Delta_{f}=\gamma_{f}^{*}, \quad \Delta_{u}=0 .
$$

Substituting the fixed point values of the regimes $(1 a)-(2 b)$ we obtain:

$$
\Delta_{f}=0 \text { for }(1 a),(2 a) ; \quad \Delta_{f}=\xi \text { for }(1 b), \quad \text { and } \quad \Delta_{f}=\xi-\eta \text { for }(2 b) \text {. }
$$

For any correlation function $G^{R}=\langle\Phi \ldots \Phi\rangle$ of the fields $\Phi$ we have $\Delta_{G}=N_{\Phi} \Delta_{\Phi}$, so the critical dimensions of the fields $\Phi=\left\{\boldsymbol{v}, \boldsymbol{\theta}, \boldsymbol{\theta}^{\prime}\right\}$ are the same as their canonical dimensions. It is the specific feature of the present model, which makes it similar to the zero-correlation time model [14] and distinguishes it from both the isotropic Kraichnan's vector model [13] (in which $\gamma_{v} \neq 0$ ) and the anisotropic Kraichnan's scalar model [7] (in which the Laplacian splitting parameter $f_{0}$ is not dimensionless). 


\section{Renormalization and critical dimensions of composite operators}

The measurable quantities are equal-time pair correlation functions of two composite fields ("operators") $F_{N p}$, built solely of the basic fields $\theta$ :

$$
G_{N_{1} p_{1}, N_{2} p_{2}}(r)=\left\langle F_{N_{1} p_{1}}\left(t, \mathbf{x}_{1}\right) F_{N_{2} p_{2}}\left(t, \mathbf{x}_{2}\right)\right\rangle, \quad F_{N p}=\left(\theta_{i} \theta_{i}\right)^{p}\left(n_{s} \theta_{s}\right)^{2 m} .
$$

Here $r=\left|\mathbf{x}_{2}-\mathbf{x}_{1}\right|$ is the separation, $N=2(p+m)$ is the total number of fields $\theta$, entering into the operator $F, i=\left\{N_{1} p_{1}\right\}$, and $k=\left\{N_{2} p_{2}\right\}$.

To calculate the renormalization constants we have to calculate the only one-loop diagram, represented in figure 3; the divergent parts of all the multiloop diagrams are equal to zero and therefore give no contribution to the renormalization constants and the anomalous dimensions.

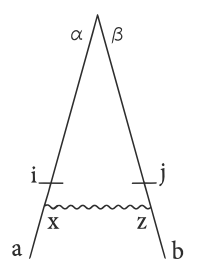

Figure 3. The one-loop contribution to the generating functional

From the calculation of this diagram it follows that the operators $F_{N p}$ indeed mix in the renormalization. This means that the renormalization constants $Z$ and the anomalous dimensions $\gamma$ are some matrices:

$$
F_{p}=\sum_{p^{\prime}} Z_{p, p^{\prime}} F_{p^{\prime}}^{R}, \quad \hat{\gamma}_{F}=\hat{Z}_{F}^{-1} \mathcal{D}_{\mu} \hat{Z}_{F}
$$

Therefore to solve the RG equation we have to diagonalize these matrices. But they appear to be not diagonalizable and can only be brought to the Jordan form.

For the equal-time pair correlation function (10) this leads to the appearance of a logarithmic dependence in the IR asymptotic behavior (in the following we denote it $G_{i k}$ for brevity):

$$
G_{i k}^{R} \propto(\mu r)^{N_{1}+N_{2}} P_{\left(N_{1}+N_{2}\right) / 2}[\ln \mu r] \Phi(1, M r, m r, \bar{f}) \quad \forall i, k .
$$

Here $P_{L}(\ldots)$ is a polynomial of degree $L$ with the argument $\ln \mu r ; \bar{f}$ is the invariant charge and $\bar{f} \rightarrow f r^{\xi}$ as $1 / \mu r \rightarrow 0$ for scaling regime (1b), $\bar{f} \rightarrow f r^{\xi-\eta}$ as $1 / \mu r \rightarrow 0$ for scaling regime (2b).

The representations (12) with unknown scaling functions $\Phi$ describe the behavior of the correlation functions at $\mu r \gg 1$ and any fixed value of $M r$. The inertial range $\ell \ll r \ll L$ corresponds to the additional condition $M r \ll 1$, the form of the functions $\Phi$ under this condition is studied using the operator product expansion.

Combining the RG representation (12) with the corresponding OPE, restoring the canonical dimension $d_{G}=-N_{1}-N_{2}$ and retaining only the leading term, we obtain the following asymptotic expression for the pair correlation function (10) in the inertial range:

$$
G=\left\langle F_{N_{1} p_{1}} F_{N_{2} p_{2}}\right\rangle \propto v^{d_{G}^{\omega}} M^{-N_{1}-N_{2}}[\ln \mu r]^{\left(N_{1}+N_{2}\right) / 2}[\ln M r]^{\left(N_{1}+N_{2}\right) / 2} \widetilde{\Phi}(\bar{f}),
$$

where $\widetilde{\Phi}(\bar{f})$ is a certain scaling function, restricted to the inertial range $\ell \ll r \ll L$. Owing to the nilpotency of the matrix of the critical dimensions, the only dependence on the exponents $\xi$ and $\eta$ that distinguishes the two nontrivial cases (1b) and (2b) from each other is contained in the invariant charge $\bar{f}$. For the trivial regimes (1a) and (2a) there is no corrections to the ordinary scaling. 


\section{Conclusion}

We applied the field theoretic renormalization group and the operator product expansion to the analysis of the inertial-range asymptotic behavior of a divergence-free vector field, passively advected by a strongly anisotropic turbulent flow. Depending on the two exponents $\xi$ and $\eta$ that describe the energy spectrum $\mathcal{E} \propto k_{\perp}^{1-\xi}$ and the dispersion law $\omega \sim k_{\perp}^{2-\eta}$ of the velocity field, the possible nontrivial types of the IR behavior appear to reduce to only two limiting cases: the rapid-change type behavior, realized for $\xi>\eta>0$ (regime $2 b$ ), and the "frozen" (time-independent or "quenched") behavior, realized for $\xi>0, \eta<0$ (regime $1 b$ ). In this respect, the situation is the same as in the model of the anisotropic advection of the scalar field studied in [7].

The inertial-range asymptotic expressions for various correlation functions are summarized by the expressions (13). In contrast to the Kraichnan's rapid-change model, where the correlation functions exhibit anomalous scaling behavior with infinite sets of anomalous exponents, here the dependence on the integral turbulence scale $L$ demonstrates a logarithmic character. The key point is that the matrices of scaling dimensions of the relevant families of composite operators appear nilpotent and cannot be diagonalized. This result is perturbatively exact in the sense that the contributions of all multiloop diagrams appear equal to zero. The physical meaning of this feature is not yet clarified, but it is clear that it is closely connected with the presence of the anisotropy vector $\mathbf{n}$.

\section{Acknowledgements}

The authors are indebted to L. Ts. Adzhemyan, Michal Hnatič, Juha Honkonen, and S. A. Paston for discussions.

The work was supported by the Saint Petersburg State University within the research grant 11.38.185.2014. N.M.G. was also supported by the Dmitry Zimin's "Dynasty" foundation and by the Saint Petersburg Committee of Science and High School.

\section{References}

[1] U. Frisch, Turbulence: The Legacy of A.N. Kolmogorov (Cambridge Univ. Pr., Cambridge, 1995)

[2] G. Falkovich, K. Gawędzki, and M. Vergassola, Rev. Mod. Phys. 73, 913 (2001)

[3] R.H. Kraichnan, Phys. Fluids 11, 945 (1968); Phys. Rev. Lett. 72, 1016 (1994)

[4] R. Grauer, J. Krug, and C. Marliani, Phys. Lett. A 195, 335 (1994); C. Salem, A. Mangeney, S.D. Bale, and P. Veltri, Astrophys. J. 702, 537 (2009); P.D. Mininni and A. Pouquet, Phys. Rev. E 80, 025401 (2009)

[5] M. Avellaneda and A. Majda, Commun. Math. Phys. 131, 381 (1990)

[6] M. Avellaneda and A. Majda, Commun. Math. Phys. 146, 139 (1992);

Q. Zhang and J. Glimm, Commun. Math. Phys. 146, 217 (1992)

[7] N.V. Antonov and A.V. Malyshev, J. Stat. Phys., 146/1, 33 (2012)

[8] O.G. Chkhetiani, M. Hnatich, E. Jurčišinova et al., Phys. Rev. E, 74, 036310 (2006)

[9] L.Ts. Adzhemyan, N.V. Antonov, A. Mazzino et al., Europhys. Lett., 55, 801 (2001)

[10] J. Zinn-Justin, Quantum Field Theory and Critical Phenomena (Clarendon, Oxford, 1989)

[11] A. N. Vasil'ev, The field theoretic renormalization group in critical behavior theory and stochastic dynamics (Chapman \& Hall/CRC, Boca Raton, 2004)

[12] N.V. Antonov, J. Phys. A: Math. Gen. 39, 7825 (2006);

N.V. Antonov and N.M. Gulitskiy, Theor. Math. Phys., 176(1), 851 (2013)

[13] N.V. Antonov and N.M. Gulitskiy, Lecture Notes in Comp. Science, 7125/2012, 128 (2012);

N.V. Antonov and N.M. Gulitskiy, Phys. Rev. E 85, 065301(R) (2012);

E. Jurčišinova and M. Jurčišin, J. Phys. A: Math. Theor., 45, 485501 (2012)

[14] N.V. Antonov and N.M. Gulitskiy, Phys. Rev. E 91, 013002 (2015)

[15] M. Hnatich, J. Honkonen, M. Jurčišin et al., Phys. Rev. E, 71, 066312 (2005)

[16] N.V. Antonov and N.M. Gulitskiy, Phys. Rev. E 92, 043018 (2015) 